\title{
Reconstruction of surface errors of the metallic waveguide based on electromagnetic scattering theory
}

\author{
$\mathrm{Na} \mathrm{Li}{ }^{*}$ and Bing Tang \\ Key Laboratory of Electronic Equipment Structure Design, \\ Ministry of Education, \\ Xidian University, \\ Xi'an, Shannxi, 710071, China \\ Email: lina@mail.xidian.edu.cn \\ Email: 281840941@qq.com \\ *Corresponding author
}

\begin{abstract}
First, the fractal model of surface errors was constructed by means of the Monte Carlo method, and the source term and spectral amplitude of surface errors were calculated. The reconstruction model of surface errors was then established. The non-differentiable problem of the fractal model was solved by the fractional differential theory. To verify the accuracy of proposed models, a waveguide filter was manufactured. The reconstruction results obtained by the fractal function model were compared with the measured results, and a comparison of the results obtained by different reconstruction models proves that the fractal function is optimal in modelling.
\end{abstract}

Keywords: reconstruction; surface error; metallic waveguide; electromagnetic scattering; fractal function.

Reference to this paper should be made as follows: Li, N. and Tang, B. (2016) 'Reconstruction of surface errors of the metallic waveguide based on electromagnetic scattering theory', Int. J. Surface Science and Engineering, Vol. 10, No. 6, pp.585-599.

Biographical notes: $\mathrm{Na} \mathrm{Li}$ is an Associate Professor at School of Mechano-electronic Engineering, Xidian University. She received her $\mathrm{PhD}$ in Mechanical Engineering from Xidian University in 2012. Her research interests include the surface error modelling theory, surface error reconstruction and the surface design.

Bing Tang is an engineer at 38th Institute of CLP group. His research interest is the reconstruction of the surface error.

\section{Introduction}

The waveguide is a fundamental part of electronic devices such as a waveguide filter, a planar slotted-waveguide array antenna, or an active phased array antenna. However, it is impossible that the inner wall of a waveguide is perfectly smooth, because of current machining technology deficits; therefore, surface errors inevitably exist (Marton and 
David, 2015; Marta et al., 2015; Xiu et al., 2014). These surface errors change the flow path of surface currents, which reduces effective surface conductivity. The overall transmission losses of a waveguide can reach up to $45 \%$ (Gu et al., 2010). Accordingly, waveguide surface errors cannot be neglected. An accurate description of surface errors is an indispensable precondition before investigating the influence mechanism of surface errors on the waveguide's transmission performance.

Generally, there are two methods used to describe a waveguide's surface errors modelling and reconstruction. Modelling is currently the predominant method (Guo et al., 2010; Ding et al., 2012). In modelling, sufficient accurate surface measurement data are required. However, after processing, the waveguide is a closed cavity, and the inner wall cannot be measured accurately. Engineers often perform measurements on the waveguide before encapsulation (Shang et al., 2011). However, during the encapsulation, new errors are likely to be produced, leading to inaccuracy of the measured data.

This may account for the low finished product ratios of these products (Nitin et al., 2014; VillaVelázquez-Mendoza et al., 2014).

There are two existing methods to reconstruct surface errors: the direct and indirect reconstruction methods (Li et al., 2015; Dong et al., 2016). In the direct method, we can directly reconstruct the contour information of errors based on the device's electrical properties (Chakrabarti et al., 2013; Cai et al., 2006). But the indirect method provides parameter information rather than the overall contour information of errors (Galdi et al., 2006; El-Shenawee et al., 2009; Xuan et al., 2013). In practical applications, the overall contour information of surface errors affects the transmission losses of a waveguide. It is not enough to have parameter information only, so the direct inversion method is the only selection for us. Desanto and Wombell (1991) innovatively introduced spectral analysis into electromagnetic scattering and inverse scattering algorithms. They substituted the source term and spectral amplitude in spectrum theory for the incident field and scattered field in electromagnetic field theory. On the basis of the reconstruction method proposed by Desanto and Wombell (1991) and Guo and Liang (2011) improved methods for solving for unknown quantities in the incident field. They adopted the mixed algorithm of the perturbation method and method of moment (MOM) to derive the Gaussian surface error on small scales; for the inversion of Gaussian surface error on large scales, they chosen the mixed algorithm of MOM and Kirchhoff approximation.

Based on these studies, we proposed a novel reconstruction method for surface errors on a waveguide's inner walls by combining the perturbation method and MOM. A cavity filter was selected for measurement and calculation, and the results calculated by the proposed method were compared with those calculated by classical models, and measured data. Our calculation results clearly fit well with the measured data and are superior to the results calculated by the classical models. 


\section{Modelling of the surface errors}

In 1975, Mandelbrot and Vannes (1968) proposed the concept of fractal, which states that similarities exist between the whole and parts. This description has gained general consent in modelling of machining surfaces. The characteristics of surface errors on waveguide's inner wall fit well with a fractal function (Liang et al., 2012; Mandelbrot, 1982). The function can be written as:

$$
f(x)=G^{D-1} \sum_{n=1}^{\infty} \frac{\cos \left(2 \pi \gamma^{n} x+\varphi_{n}\right)}{\gamma^{(2-d) n}},(1<D<2)
$$

in which, $D$ denotes the fractal dimension, $\gamma$ denotes the spatial frequency, and $\gamma>1 . n$ is the number of tones and $\varphi_{n}$ is a phase term that had a uniform distribution over the interval $[-\pi, \pi]$. Figure 1 presents the one-dimensional surface error models based on the W-M fractal function with different amplitudes $(G)$, when $\gamma=1.5$ and $D=1.3$.

The two curves in Figure 1 show that when the fractal dimension $D$ and $\gamma$ remain unchanged and the value of amplitude $G$ varies, the two models exhibit similar height fluctuations frequencies and local fine structures. However, the heights of the fluctuations of these two models are slightly different, suggesting that the value of $G$ is mainly used for controlling the amplitude of surface errors.

Figure 1 One-dimensional surface error models with different amplitudes (see online version for colours)

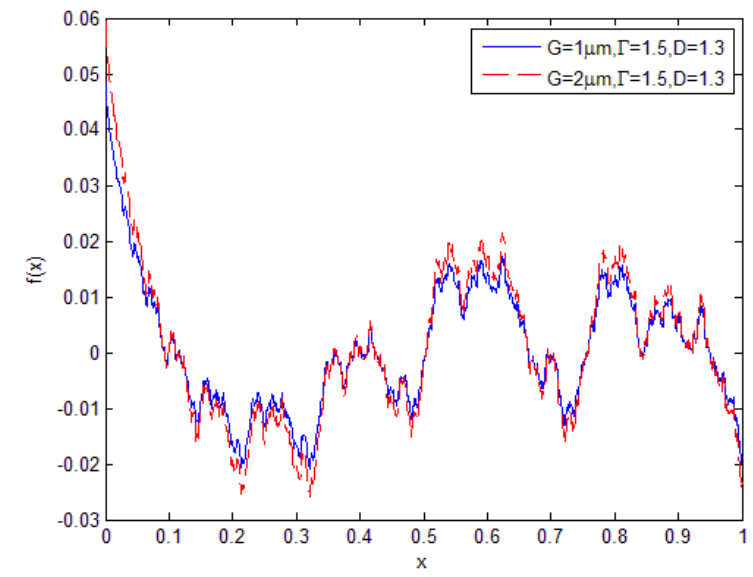


Figures 2(a) and 2(b) show that when the values of $G$ and $\gamma$ are identical, these two models exhibit differences not only in terms of amplitude, but also in local fine structures. Moreover, the larger the value of fractal dimension $D$, the local fine structures are more complicated. Therefore, we can conclude that fractal dimension $D$ reflects the degree of complexity, irregularity, fineness, and filling of surface errors in the space. Specifically, a larger value of fractal dimension $D$ is indicative of higher complexity.

Figure 2 One-dimensional roughness models with different fractal dimensions

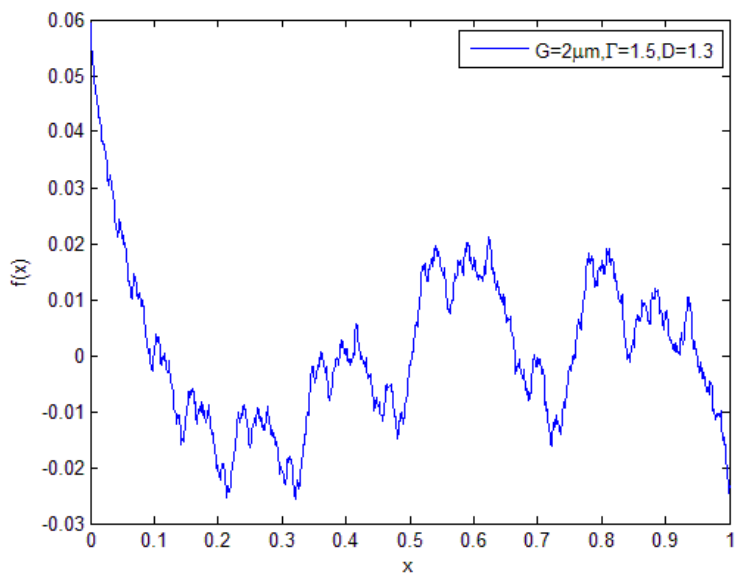

(a)

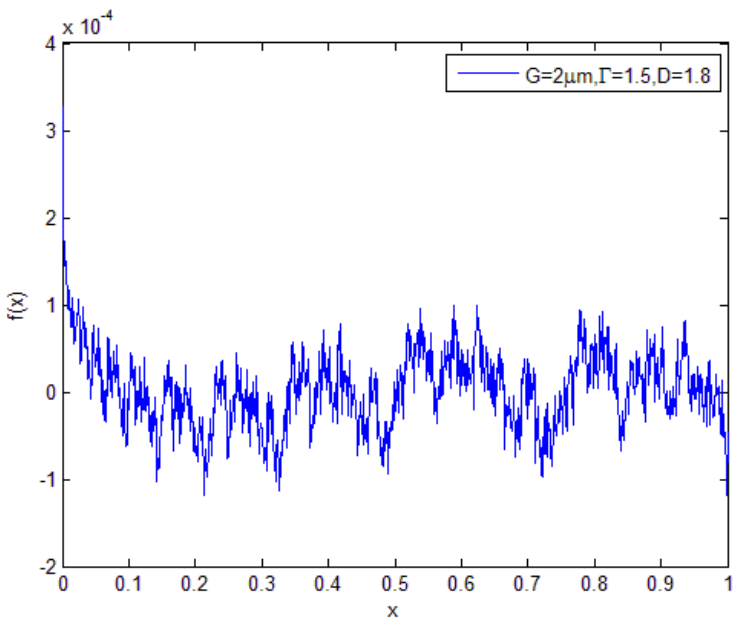

(b)

By comparing the two curves in Figures 3(a) and 3(b), we can conclude that when the values of $G$ and $D$ are same, the parameter of $\gamma$ mainly affects the overall smoothness of the error models. The closer the value of $\gamma$ approaches 1 , the smoother the model is. In some extreme cases when $\gamma=1$, as described in equation (1), the fractal error function can be written as the summation of a series of periodic functions, and the model can be transformed into a smooth curve. Therefore, for a fractal model, the value of $\gamma$ must be larger than 1 . 
Figure 3 One-dimensional roughness models with different fractal frequencies (see online version for colours)

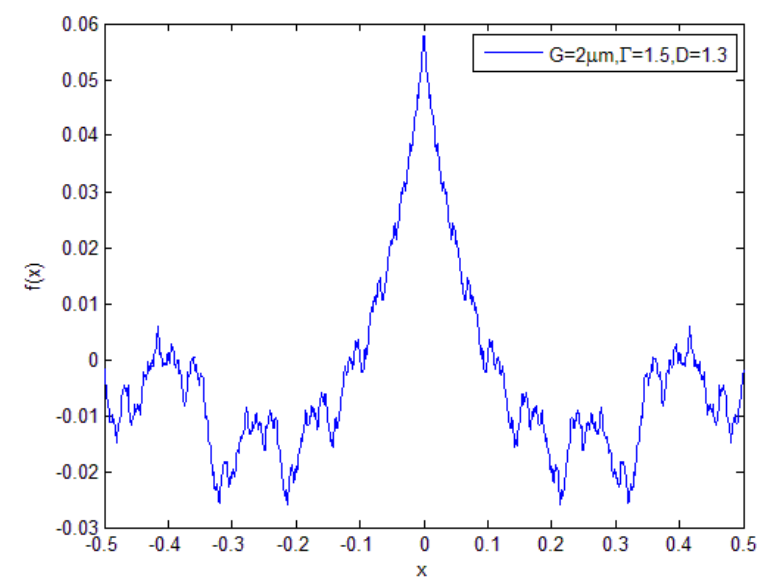

(a)

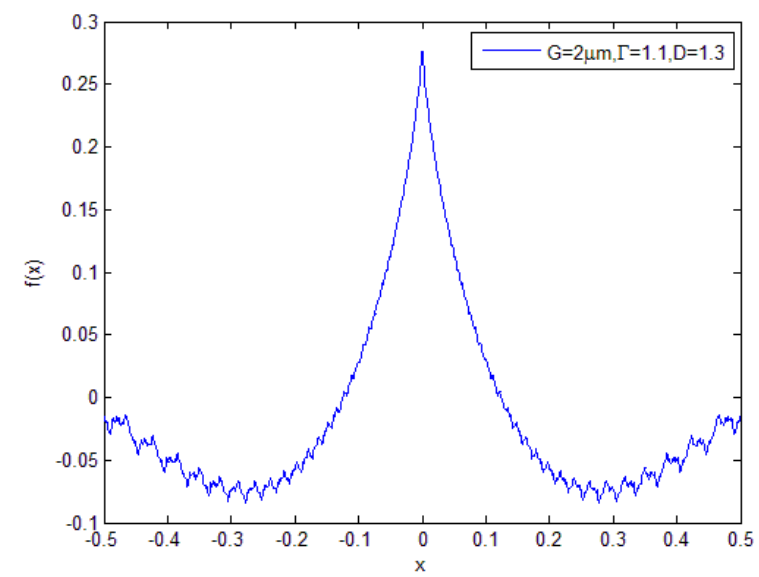

(b)

\section{Reconstruction of the surface errors}

\subsection{Source term and spectral amplitude of surface errors}

The sampling length of surface errors was set as $L$, and then a series of discrete points were selected within the range from $\frac{-L}{2}$ to $\frac{L}{2}$, i.e., $\frac{-L}{2} \leq x \leq \frac{L}{2}$. When $|x|>\frac{L}{2}$, the surface current of errors is zero, and the edge current on the surface of errors suddenly becomes zero from non-zero values, giving rise to an artificial reflection. To avoid this problem, the pyramidal wave was selected as the illumination source. From the central point of error surface to both ends, the exposure intensity of the pyramidal wave was gradually attenuated to be zero in the form of Gaussian function. The pyramidal wave 
function can be written as (Tsang et al., 2001):

$$
\varphi_{i}(x, z)=e^{i k_{0}\left(x \sin \theta_{i}-z \cos \theta_{i}\right)(1+W)} \times e^{-\left(\frac{x+z \tan \theta_{i}}{g}\right)^{2}}
$$

in which $k 0$ is the wave number, $g$ refers to the pyramidal factor and represents the beam length of the pyramidal wave, $\theta_{i}$ denotes the incident angle, and $W$ is the additional phase term. This can be written as:

$$
W=\frac{2 \frac{\left(x+z \tan \theta_{i}\right)^{2}}{g^{2}}}{\left(k_{0} g \cos \theta_{i}\right)^{2}} .
$$

In 1985, US researcher Desanto proposed a method to solve the electromagnetic scattering problem for rough surfaces. This method is called the spectral representation method, whose basic ideas are described below. When the Dirichlet boundary condition is satisfied, the integral equations, which include the morphology function of the rough surface, $f(x)$, can be expressed in frequency domain. These two integral equations are referred to as the source term and spectral amplitude. Their mathematical forms can be written as (Liang et al., 2011):

$$
\begin{aligned}
& R_{i}(q, p)=\int_{-\infty}^{\infty} \varepsilon(x, p) e^{-i q x} e^{i \alpha(q) f(x)} d x \\
& R_{s}(q, p)=\int_{-\infty}^{\infty} \varepsilon(x, p) e^{-i q x} e^{-i \alpha(q) f(x)} d x
\end{aligned}
$$

in which $R_{i}(q, p)$ denotes source term, $R_{s}(q, p)$ denotes the spectral amplitude of the scattered field, $p$ and $q$ are the incident and scatter wave vectors, respectively, and $p=k_{0} \sin \theta_{i}, k_{0}=\frac{2 \pi}{\lambda}, q=k_{0} \sin \theta_{s}, \alpha(q)=k_{0} \sqrt{1-q^{2}}=k_{0} \cos \theta_{s}, \theta_{i}$ denotes the incident angle, and $\theta_{s}$ denotes the scattering angel. $\varepsilon(x, p)$ is a scalar with no units and exhibits the following relationship with the total field of error surface:

$$
\varepsilon(x, p)=\left(i k_{0}\right)^{-1}\left(\partial_{z}-f^{\prime}(x) \partial_{n}\right) \times\left.[\varphi(x, z)]\right|_{z=f(x)}
$$

in which $\partial_{z}$ denotes the partial derivative operator along the $z$ direction, and $\partial_{n}$ denotes a partial derivative operator along the normal direction.

At any distance above the surface, where $z=h$, the relationship between the source term and the incident field can be expressed as (Liang et al., 2011):

$$
R_{i}(q, p)=e^{i \alpha(q) h} \int_{-\infty}^{\infty}\left[\frac{\varphi_{i}(x, h) \alpha(p)}{k_{0}}-M(x, h)\right] \times e^{-i q x} d x
$$

in which $\alpha(p)=k_{0} \sqrt{1-p^{2}}=k_{0} \cos \theta_{i}$ and $M=\left(i k_{0}\right)^{-1} \partial_{z} \varphi_{i}$.

The spectral amplitudes for the far field and scattered fields have the following relationship: 


$$
R_{s}(q, p)=\lim _{k r \rightarrow \infty} \varphi_{s}(x, z) \sqrt{\frac{2 k}{\pi r}} e^{i k r} e^{\frac{i \pi}{4}}
$$

in which, $r=\sqrt{x^{2}+z^{2}}$.

\subsection{Reconstruction model of surface errors}

Based on Euler's formula $e^{ \pm i x}=\cos (x) \pm i \sin (x)$, the source term and spectral amplitude can be written as:

$$
\begin{aligned}
& R_{i}(q, p)=\int_{-\infty}^{\infty} \varepsilon(x, p) e^{-i q x}\{\cos (\alpha(q) f(x))+i \sin (\alpha(q) f(x))\} d x \\
& R_{s}(q, p)=\int_{-\infty}^{\infty} \varepsilon(x, p) e^{-i q x}\{\cos (\alpha(q) f(x))-i \sin (\alpha(q) f(x))\} d x
\end{aligned}
$$

Let $\Omega_{+}(q, p)=\frac{R_{i}(q, p)+R_{s}(q, p)}{2}$ and $\Omega_{-}(q, p)=\frac{i R_{s}(q, p)-i R_{i}(q, p)}{2}$, combined with equations (6) and (7), we can obtain:

$$
\begin{aligned}
& \Omega_{+}(q, p)=\int_{-\infty}^{\infty} \varepsilon(x, p) e^{-i q x} \cos (\alpha(q) f(x)) d x \\
& \Omega_{-}(q, p)=\int_{-\infty}^{\infty} \varepsilon(x, p) e^{-i q x} \sin (\alpha(q) f(x)) d x
\end{aligned}
$$

For the surface errors on small scales, $|\alpha(q) f(x)| \ll 1$, approximately, $\alpha(q) f(x)=0$. The product factors can be written as $\cos (\alpha(q) f(x))=1$ and $\sin (\alpha(q) f(x))=\alpha(q) f(x)$. Given these two assumed conditions, the solution of the inversion model $f^{\prime}(x)$ of surface errors can be expressed as:

$$
f^{\prime}(x)=\frac{F_{-}(x)}{F_{+}(x)}=\frac{F^{-1}\left[\frac{\Omega_{-}(q, p)}{\alpha(q)}\right]}{F^{-1}\left[\Omega_{+}(q, p)\right]}
$$

in which $F-1(\cdot)$ denotes Fourier inversion.

The series of inversion formulas stated above allow us to conclude that, to solve the reconstruction problem of one-dimensional surface errors on small scales, the source term and spectral amplitudes should be first combined and approximated (He et al., 2014). Then the Fourier inversion can be conducted on these data, and the surface errors can be reconstructed. This method is called the mixed reconstruction algorithm of MOM and the perturbation method (abbreviated as the MON-SPA method). 


\subsection{Reconstruction of fractal model}

For each side of the fractal model equation (1), we take the derivative with respect to $x$,

$$
f^{\prime}(x)=-2 \pi G^{D-1} \sum_{n=1}^{\infty} \frac{\sin \left(2 \pi \gamma^{n} x\right)}{\gamma^{(1-D) n}}
$$

It can be found that, when $1<D<2$, the derivative $f^{\prime}(x)$ approaches infinity, i.e., $f(x)$ is non-differentiable, and its derivative does not exist.

Since each point in the fractal function is continuous and non-differentiable, it becomes fairly difficult to solve source term and spectral amplitude. To give an accurate expression, we should first take the derivative of the fractal function. However, each point in fractal function is non-differentiable; $\varepsilon(x, p)$ cannot be accurately expressed and the subsequent inversion process cannot move forward.

Regarding the fractional calculus for the fractal function, the scholars have performed many studies, which have also been successfully applied in several aspects. For example, Mandelbort and Salvatore applied fractional calculus to processing white noises and images and acquired some desirable results (Enriquez et al., 2004; Salvatore and Mario, 2014). In China, Yao and Zhou conducted in-depth studies on the characteristics of the fractional calculus image for the fractal function. They discussed the fractal dimension of the fractional calculus images in detail (Liang and $\mathrm{Su}, 2008$ ). Based on fractal theory and fractional calculus theory, the derivative of fractal function can be determined as follows. First, the fractional calculus in fractal geometry can be defined as:

$$
D^{-v} f(x)=\frac{1}{\Gamma(v)} \int_{-\infty}^{x}(x-t)^{v-1} f(t) d t
$$

in which $\Gamma(v)=\int_{-\infty}^{\infty} e^{-t} t^{v-1} d t$ is the Gamma function. $D^{-v} f(x)$, in the form of fractional calculus, can be referred to as the -order integral function of $f(x)$. The parameter $\mu$ is then introduced, $0<\mu<1, \mu=1-v$, and we can obtain the following expression:

$$
D^{\mu} f(x)=D\left[D^{-v} f(x)\right]
$$

$D^{-v} f(x)$, in the form of fractional calculus, is then referred to as the $\mu$-order differentiation function of $f(x)$.

According to these definitions, the fractional calculus expression of W-M fractal function, denoted as $g(x)$, can be derived and written as:

$$
g(x)=D^{\mu} f(x)=G^{D-1} \sum_{n=1}^{\infty} \gamma^{(D-1) n} S_{x}\left(1-\mu, 2 \pi \gamma^{n}\right)
$$

in which the function $S_{x}\left(1-\mu, 2 \pi \gamma^{n}\right)$ is the $\mu$-order integral form of $\sin \left(2 \pi \gamma^{n} x\right)$. It can be mathematically expressed as:

$$
S_{x}\left(1-\mu, 2 \pi \gamma^{n}\right)=D^{\mu} \sin \left(2 \pi \gamma^{n} x\right)=\frac{1}{\Gamma(\mu)} \int_{-\infty}^{x}(x-t)^{\mu-1} \sin \left(2 \pi \gamma^{n} t\right) d t
$$


To invert the fractal model, we first solve the fractional derivative of W-M fractal function. The function $\varepsilon(x, p)$ can then be solved.

Figure 4 Source terms of fractal surface errors with different incident angles (see online version for colours)

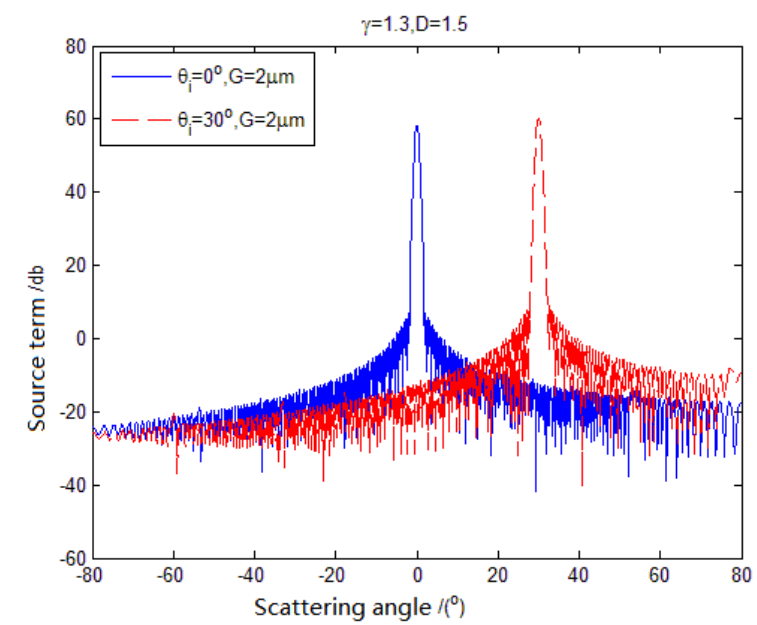

As shown in Figure 4, for one-dimensional fractal surface errors, the source terms reach the peaks at the scattered angles $\left(0^{\circ}\right.$ and $\left.30^{\circ}\right)$ and then decrease toward two sides that are irrelevant to the errors' height information. The results are compatible with the conclusions for source terms in Gaussian surface error and periodic surface error, which suggests that it is reasonable to use the fractional derivative model to calculate the source term of fractal surface errors.

Figure 5 Spectral amplitudes of fractal surface errors with different incident angles (see online version for colours)

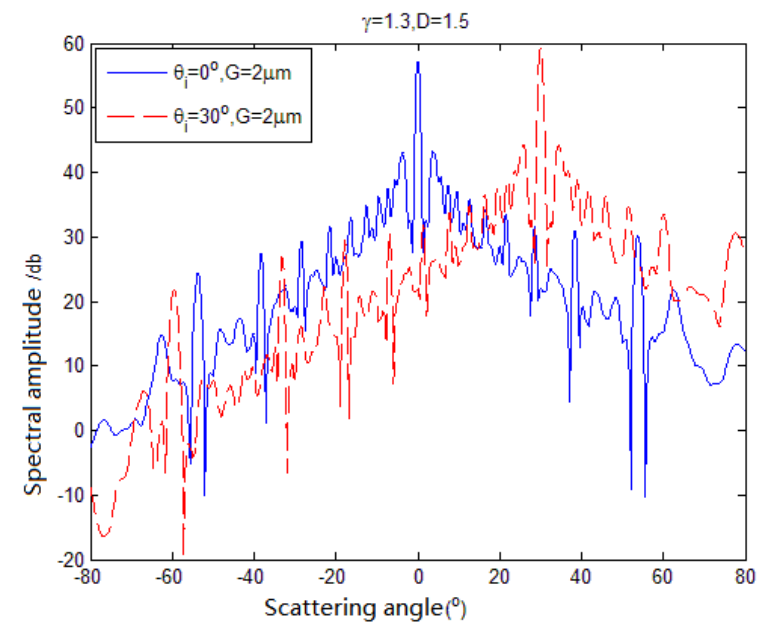


As shown in Figure 5, for one-dimensional fractal surface errors, the spectral amplitudes are larger at the middle position of $\mathrm{x}$-axis and smaller on both sides. When the incident angles are $0^{\circ}$ and $30^{\circ}$, the spectral amplitudes reach the peaks at their respective mirroring scattered angles and decline gradually toward the sides. These results are also consistent with the spectral amplitudes in Gaussian surface error and periodic surface error, suggesting that calculating the spectral amplitudes of fractal surface errors using fractional derivative model is also reasonable.

\section{Experimental verification of proposed methods}

\subsection{Introductions the sample and experiment condition}

To verify the accuracy of the proposed reconstruction method, a seven-stage waveguide filter as shown in Figure 6(a) was selected and the surface errors on its upper inner walls were measured. Errors are measured by the Taylor Hobson profile measuring instrument, and the concrete testing parameters are $\mathrm{R} / 4 * 0.8 \mathrm{~mm} / \mathrm{G} / 300 / \mathrm{LS}$ and $4.1 \mathrm{~mm} /$ Admin/INTRA $50 \mathrm{~mm}$. Figure 6(b) presents the measured surface errors of the waveguide filter. The reconstruction model based on the fractal function was used for a direct comparison.

Figure 6 (a) A seven-stage waveguide filter (b) Measured surface errors of the inner walls of a cavity filter (see online version for colours)

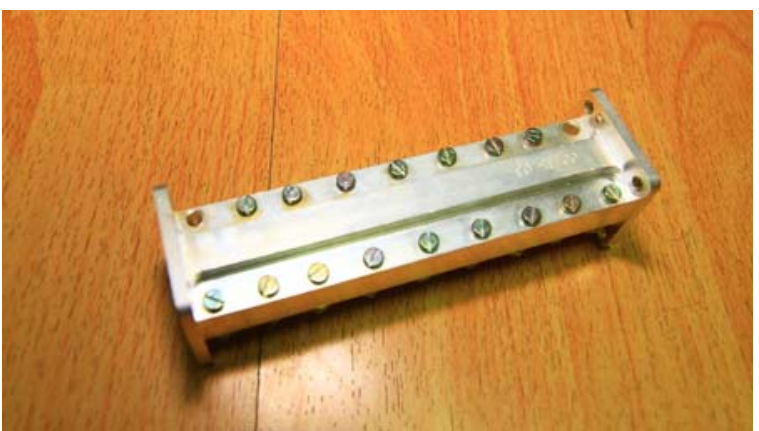

(a)

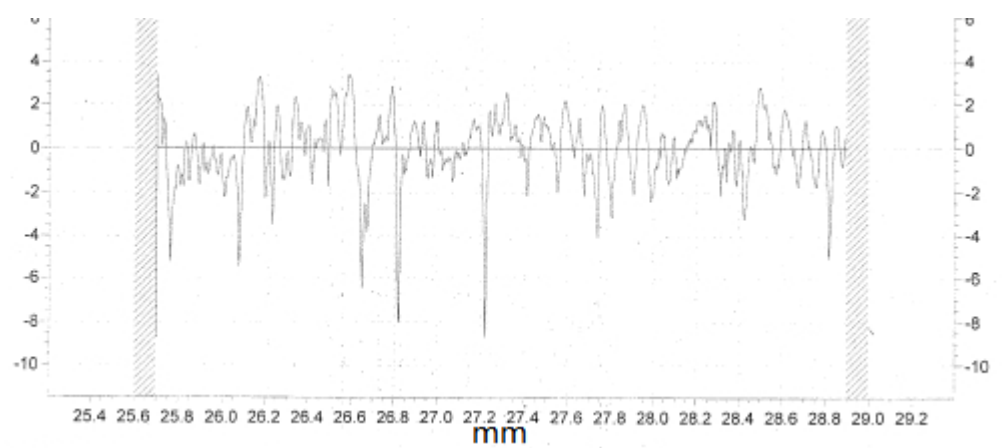

(b) 
The local contour of these measured results was reconstructed with the fractal function. Figure 7 shows the measured data and the data calculated by reconstruction model for a direct comparison.

Figure 7 Comparison of the measured data and reconstruction model (see online version for colours)

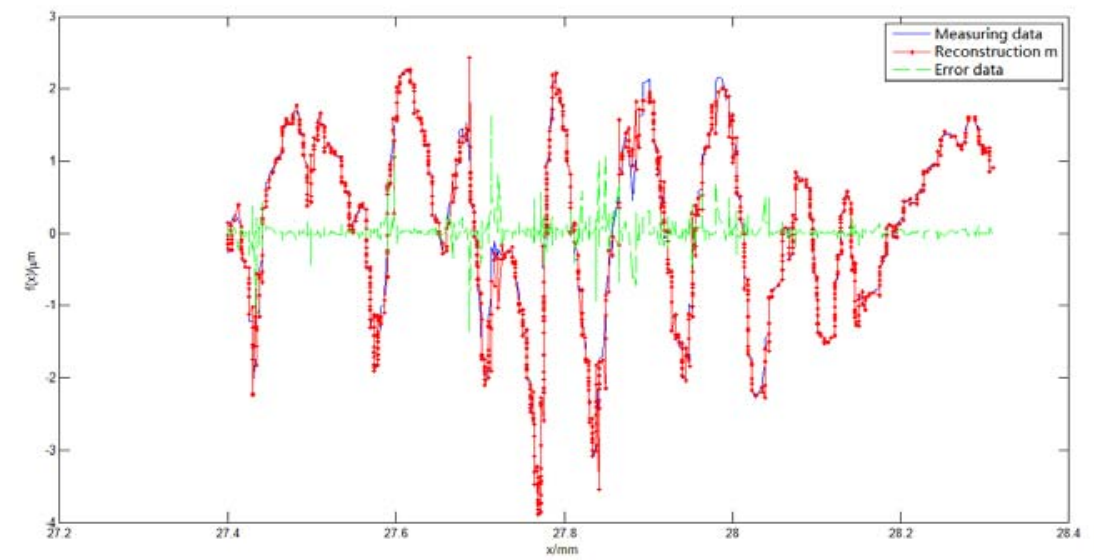

Figure 7 shows that the data calculated by the reconstruction model are in good agreement with the measured data, with an error value of $0.0071 \mu \mathrm{m}\left(1.578 \times 10^{-5} \lambda\right)$. Subsequently, in order to verify the superiority of the proposed method, the reconstruction calculations were conducted on the same error surface using the exponential function, the Gaussian function, and the fractal function. Then the error values of the various reconstruction models were compared. The exponential function, the Gaussian function, and the fractal function were denoted by $f_{1}\left(\delta_{1}, l_{1}\right), f_{2}\left(\delta_{2}, l_{2}\right)$ and $f_{3}(G, D)$, respectively, in which $\delta$ and $l$ denotes the root-mean-square height and correlation length, respectively, $G$ denotes the amplitude of fractal model, and $D$ denotes the fractal dimension. In terms of the power spectral density function of the error surface, the power spectral density functions of the exponential function and the Gaussian function can be expressed as (Xu et al., 2012; Elson and Bennett, 1995):

$$
\begin{aligned}
& p_{1}(\omega)=\frac{\delta_{1}^{2} l_{1}}{\pi\left(1+\omega^{2} l_{1}^{2}\right)} \\
& p_{2}(\omega)=\frac{\delta_{2}^{2} l_{2}}{2 \sqrt{\pi}} e^{\left(\frac{-\omega^{2} l_{2}^{2}}{4}\right)}
\end{aligned}
$$

The power spectral density function for the error surface described by W-M fractal function can be obtained by discrete Fourier transformation (Zhou and He, 2004):

$$
p(\omega)=F\left(f\left(x_{i}\right)\right)
$$


After transformation, we took the logarithm of the result and acquired the curve of $\log p(\omega) \sim \log (\omega)$. Linear fitting was performed on the curve using the least square method. After the line of best fit was found, the slope and intercept exhibited the following relationship with the primary parameter of fractal function $(G$ and $D)$ :

$$
\begin{aligned}
& D=\frac{5+k}{2} \\
& B=2(D-1) \log G-\log (2 \ln \gamma)
\end{aligned}
$$

in which $k$ and $B$ denote the slope and intercept of the straight line after fitted. The amplitude $G$ and fractal dimension $D$ of the fractal function can also be solved. As shown in Figure 8, the power spectrum curve and the fitting line of the reconstruction W-M show a good agreement with the test data, and the error on the indexes value of test data $\left(k_{1}\right.$ and $\left.B_{1}\right)$ and that $\mathrm{W}-\mathrm{M}$ function $\left(k_{2}\right.$ and $\left.B_{2}\right)$ is small.

Figure 8 Comparison of the power spectrum curve and fitting line of the W-M to test data (see online version for colours)

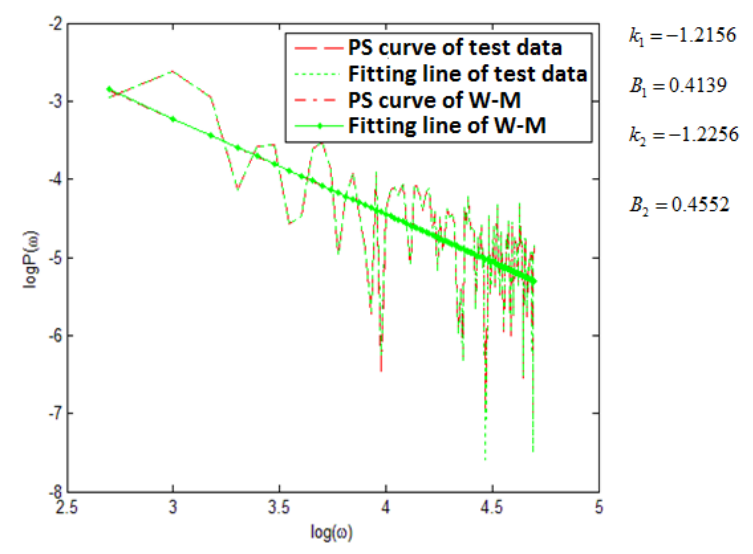

Figure 9 Reconstruction results of the error surface using different model functions (see online version for colours)

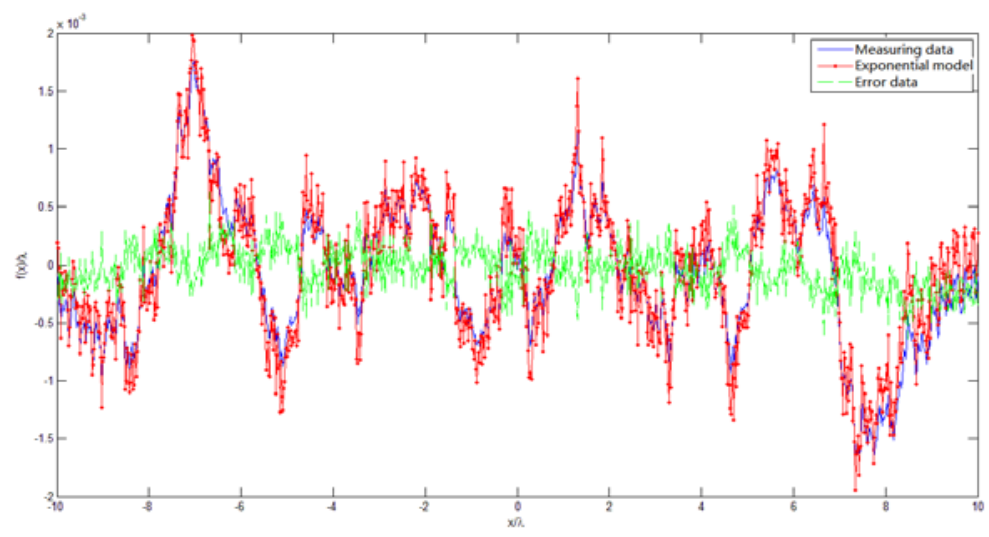

(a) 
Figure 9 Reconstruction results of the error surface using different model functions (continued) (see online version for colours)

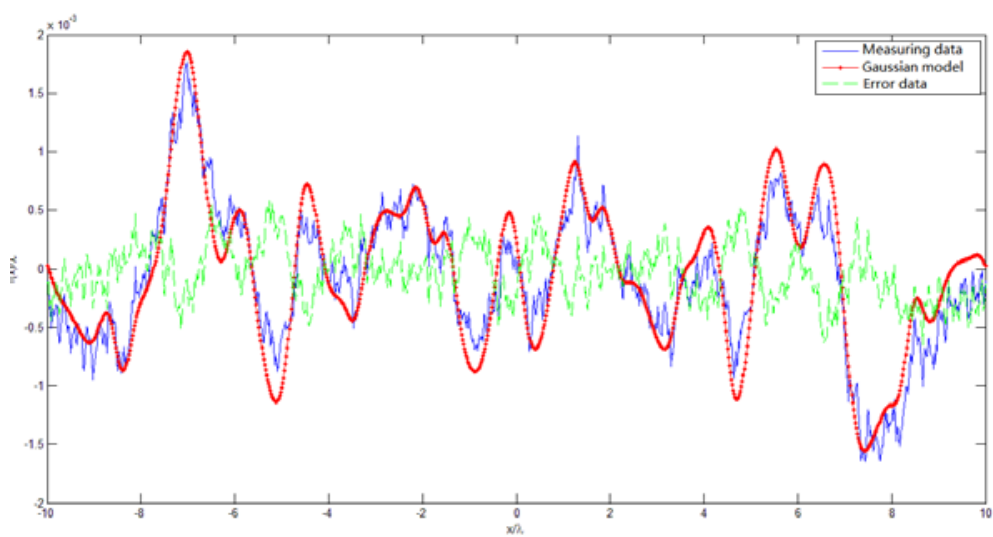

(b)

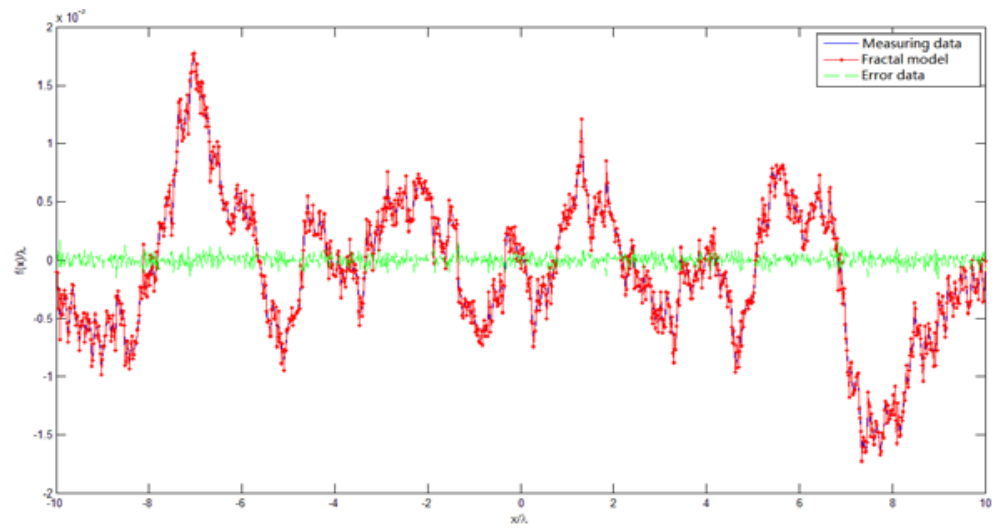

(c)

\subsection{Results and discussion}

As shown in Figures 9(a)-9(c), the error values based on these three reconstruction models are $1.0170,2.7040$, and 0.4331 , respectively. When the fractal model is being used, the error value is at the minimum and the reconstruction results are optimal.

\section{Conclusions}

The reconstruction problem for scattering at grazing incidence by one-dimensional surfaces has been reformulated as a pair of coupled integral equations, relating the unknown surface derivative and the value of the scattered field at two lines in the medium. The surface errors were modelled by a one-dimensional fractal function. Based on the error model, source term and spectral amplitude of surface errors were calculated. The reconstruction model of surface errors was then established using the mixed 
algorithm of the perturbation method and the MOM. To validate the effectiveness of reconstruction equations, a waveguide filter was manufactured. The results are based on comparison with test data of the filter. It is clear that surface errors have the fractal characteristic, the fractal model of surface errors more accuracy than the exponential and Gaussian model. The fractal reconstruction model is well agreed with the measured data.

\section{Acknowledgements}

The authors would like to thank the financial support of the National Natural Science Foundation of China (No: 51305322; 51490660).

\section{References}

Cai, Z.J. et al. (2006) 'Reconstruction of a fractal rough surface', Physica D: Nonlinear Phenomena, Vol. 213, No. 1, pp.25-30.

Chakrabarti, S. et al. (2013) 'Reconstruction of the surface-height autocorrelation function of a randomly rough dielectric surface from incoherent light scattering', Physical Review, Vol. 88, No. 1, p.083812-1-6.

Desanto, J.A. and Wombell, R.J. (1991) 'Reconstruction of rough-surface profiles with the Kirchhoff approximation', Journal of the Optical Society of America A-Optics Image Science and Vision, Vol. 8, No. 12, pp.1892-1897.

Ding, R.H. et al. (2012) 'Wave propagation in parallel plate metallic waveguide with finite conductivity and three dimensional roughness', IEEE Transactions on Antennas and Propagation, Vol. 60, No. 3, pp.5867-5880.

Dong, H.P. et al. (2016) 'Inverse electromagnetic scattering for a locally perturbed perfectly conducting plate', Wave Motion, Vol. 60, No. 1, pp.95-107.

El-Shenawee, M. et al. (2009) 'An adjoint-field technique for shape reconstruction of 3-D penetrable object immersed in lossy medium', IEEE T. Antennas Propag., Vol. 57, No. 2, pp.520-534.

Elson, J.M. and Bennett, J.M. (1995) 'Calculation of the power spectral density from surface profile data', Applied Optics, Vol. 34, No. 1, pp.201-208.

Enriquez, N. et al. (2004) 'A simple construction of the fractional Brownian motion', Stochastic Processes and their Applications, Vol. 109, No. 2, pp.203-223.

Galdi, V. et al. (2006) 'Short-pulse three-dimensional scattering from moderately rough surfaces: a comparison between narrow-waisted Gaussian beam algorithms and FDTD', IEEE Transactions on Antennas and Propagation, Vol. 54, No. 1, pp.157-167.

$\mathrm{Gu}, \mathrm{X} . \mathrm{X}$. et al. (2010) 'Modelling effects of random rough interface on power absorption between dielectric and conductive medium in 3-D problem', IEEE Transactions on Microwave Theory and Techniques, Vol. 55, No. 3, pp.511-517.

Guo, L.X. and Liang, Y. (2011) 'A study of electromagnetic scattering from conducting targets above and below the dielectric rough surface', Optics. Express, Vol. 19, No. 7, pp.5785-5801.

Guo, X.C. et al. (2010) 'An analysis of conductor surface roughness effects on signal propagation for stripline interconnects', IEEE Transactions on Electromagnetic Compatibility, Vol. 56, No. 5, pp.707-714.

He, Y. et al. (2014) 'A new spectral method for numerical solution of the unbounded rough surface scattering problem', Journal of Computational Physics, Vol. 275, No. 7, pp.608-625.

Li, J.L. et al. (2015) 'A nonlinear integral equation method for the inverse scattering problem by sound-soft rough surfaces', Inverse Problems in Science and Engineering, Vol. 23, No. 4, pp.557-577. 
Liang, X. et al. (2012) 'Fractal analysis of engineering ceramics ground surface', Applied Surface Science, Vol. 258, No. 17, pp.6406-6015.

Liang, Y. et al. (2011) 'Investigation on the reconstruction of rough surface with hybrid method', Acta Physica Sinica, Vol. 60, No. 3, pp.1-9.

Liang, Y.S. and Su, W.Y. (2008) 'The relationship between the Box dimension of the Besicovitch functions and the orders of their fractional calculus', Applied Mathematics and Computation, Vol. 200, No. 1, pp.297-307.

Mandelbrot, B.B. (1982) The Fractal Geometry of Nature, Freeman, New York.

Mandelbrot, B.B. and Vannes, J.W. (1968) 'Fractional Brownian motions, fractional noise and applications', Siam Review, Vol. 10, No. 5, pp.422-437.

Marta, H. et al. (2015) 'Measurement and assessment of surface roughness for optimisation of laser cutting technology', Int. J. of Surface Science and Engineering, Vol. 9, Nos. 2-3, pp.264-279.

Marton, H. and David, T.G. (2015) 'Surface characterisation of electrical discharge pulse-mode textured cold work rolls', Int. J. of Surface Science and Engineering, Vol. 9, No. 4, pp.328-342.

Nitin, S. et al. (2014) 'Investigations on the effects of surface texture on the performance of a porous journal bearing operating with couple stress fluids', Int. J. of Surface Science and Engineering, Vol. 8, No. 4, pp.392-407.

Salvatore, B. and Mario, D.P. (2014) 'A physically based connection between fractional calculus and fractal geometry', Annals of Physics, Vol. 350, No. 11, pp.146-158.

Shang, X. et al. (2011) 'Micromachined W-band waveguide and filter with two embedded H-plane bends', IET Microwaves, Antennas \& Propagation, Vol. 5, No. 3, pp.334-339.

Tsang, L., Ak, J. and Ding, K.H. (2001) Scattering of Electromagnetic Waves: Numerical Simulations, Wiley Interscience, New York.

VillaVelázquez-Mendoza, C.I. et al. (2014) 'Effect of substrate roughness, time and temperature on the processing of iron boride coatings: experimental and statistical approaches', Int. J. of Surface Science and Engineering, Vol. 8, No. 1, pp.71-91.

Xiu, S.C. et al. (2014) 'Study on micro-surface texture and tribology characters of ground surface in point grinding process', Int. J. of Surface Science and Engineering, Vol. 8, Nos. 2-3, pp.225-238.

Xu, C. et al. (2012) 'Topographic power spectral density study of the effect of surface treatment processes on niobium for superconducting radio frequency accelerator cavities', Physical Review Special Topics - Accelerators and Beams, Vol. 15, No. 4, pp.1-6.

Xuan, Y.M. et al. (2013) 'Reconstruction of two-dimensional randomly rough surfaces based on bidirectional reflectance distribution function', International Journal of Thermophysics, Vol. 34, No. 3, pp.450-466.

Zhou, S. and He, G. (2004) 'On a class of fractals: the constructive structure', Chaos Solitons \& Fractals, Vol. 19, No. 5, pp.1099-1104. 